\title{
Structure of 'Linkerless' Hydroxamic Acid Inhibitor-HDAC8 Complex Confirms the Formation of an Isoform-Specific Subpocket
}

\author{
Alexa A. Tabackman, ${ }^{a}$ Rochelle Frankson, ${ }^{b}$ Eric S. Marsan, ${ }^{a}$ Kay Perry, ${ }^{c}$ and Kathryn E. Cole*a \\ ${ }^{a}$ Department of Molecular Biology and Chemistry, Christopher Newport University, 1 Avenue of \\ the Arts, Newport News, VA, 23606 \\ ${ }^{\mathrm{b}}$ Department of Chemistry, Ithaca College, 950 Danby Road, Ithaca, NY, 14850 \\ ${ }^{\mathrm{c}}$ Northeastern Collaborative Access Team (NE-CAT) and Department of Chemistry and \\ Chemical Biology, Cornell University, Building 436E, Argonne National Laboratory, 9700 South \\ Cass Avenue, Argonne, IL 60439, USA \\ *To whom correspondence should be addressed: kathryn.cole@ cnu.edu
}

\section{Keywords}

Histone deacetylase 8; Histone deacetylase inhibitors (HDACi); hydroxamic acids; AutoDock Vina

The atomic coordinates and structure factors of the HDAC8-inhibitor 6 complex have been deposited in the Protein Data Bank (www.rcsb.org) with accession code 5FCW. 


\begin{abstract}
Histone deacetylases (HDACs) catalyze the hydrolysis of acetylated lysine side chains in histone and non-histone proteins, and play a critical role in the regulation of many biological processes, including cell differentiation, proliferation, senescence, and apoptosis. Aberrant HDAC activity is associated with cancer, making these enzymes important targets for drug design. In general, HDAC inhibitors (HDACi) block the proliferation of tumor cells by inducing cell differentiation, cell cycle arrest, and/ or apoptosis, and comprise some of the leading therapies in cancer treatments. To date, four HDACi have been FDA approved for the treatment of cancers: suberoylanilide hydroxamic acid (SAHA, Vorinostat, Zolinza $\left.{ }^{\circledR}\right)$, romidepsin (FK228, Istodax $\left.^{\circledR}\right)$, belinostat $\left(\right.$ Beleodaq $\left.^{\circledR}\right)$, and panobinostat $\left(\right.$ Farydak $\left.^{\circledR}\right)$. Most current inhibitors are panHDACi, and non-selectively target a number of HDAC isoforms. Six previously reported HDACi were rationally designed, however, to target a unique sub-pocket found only in HDAC8. While these inhibitors were indeed potent against HDAC8, and even demonstrated specificity for HDAC8 over HDACs 1 and 6, there were no structural data to confirm the mode of binding. Here we report the X-ray crystal structure of Compound 6 complexed with HDAC8 to $1.98 \AA$ resolution. We also describe the use of molecular docking studies to explore the binding interactions of the other 5 related HDACi. Our studies confirm that the HDACi induce the formation of and bind in the HDAC8-specific subpocket, offering insights into isoform-specific inhibition.
\end{abstract}

\title{
Introduction
}

Histone deacetylases (HDACs) catalyze the hydrolysis of acetylated lysine side chains in histone and non-histone proteins, and play a critical role in the regulation of many biological 
processes, including cell differentiation, proliferation, senescence, and apoptosis (De Ruijter et al., 2003; Emanuele et al., 2008; Haberland et al., 2009). Aberrant HDAC activity is associated with various cancers, where increased HDAC activity has been linked to the silencing of tumor suppressor genes (Glozak and Seto, 2007). As such, HDACs are validated targets for drug design. HDAC inhibitors (HDACi) function by obstructing the proliferation of tumor cells by inducing cell differentiation, cell cycle arrest, and/or apoptosis, and are some of the leading therapies for the treatment of cancer (Mei et al., 2004; Bolden et al., 2006). Favorably, some HDACi exhibit a low toxicity against normal cells, unlike other available cancer therapeutics (Huang and Pardee, 2000).

To date, the FDA has formally approved four compounds, and a number of other HDACi are currently in clinical trials. The first compound to be approved was Vorinostat, also known as suberoylanilide hydroxamic acid (SAHA) and marketed as Zolinza (Merck), in 2006. SAHA is a second-generation hydroxamate inhibitor that was originally approved for the treatment of cutaneous T-cell lymphoma (CTCL) (Mann et al., 2007). It is currently in both mono- and hybrid-therapy clinical trials for the treatment of prostate, pancreatic, and non-small cell lung cancers, in addition to various lymphomas and forms of leukemia (http://clinicaltrials.gov/). Romidepsin (Istodax ${ }^{\circledR}$, Celgene) is a cyclic tetrapeptide that was approved in 2009 also for the treatment of CTCL, and is currently in clinical trials for the treatment of other lymphomas (Guan

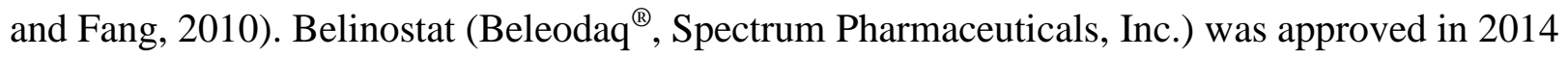
for the treatment of relapsed peripheral T-cell lymphoma (Lee et al., 2015), and the most recent drug, Panobinostat (Farydak ${ }^{\circledR}$, Novartis), was approved in 2015 for the treatment of relapsed multiple myeloma (Fenichel, 2015). 
HDACi typically employ a pharmacophore model that includes: a zinc binding group to bind and inhibit the catalytic zinc ion, a linker segment to mimic substrate binding and occupy the active site tunnel, and a hydrophobic 'cap group' to interact with the surface residues lining the active site pocket (Figure 1A). X-ray crystal structures of hydroxamic acid HDACi complexed with HDAC8 provide insight into the specific enzyme-inhibitor interactions that are essential for drug design (Somoza et al., 2004; Vannini et al., 2004; Vannini et al., 2007; Dowling et al., 2008; Dowling et al., 2010). In all structures, the hydroxamate moiety binds to the catalytic zinc ion in the active site and completes the square pyramidal coordination geometry of the metal ion (Figure 1B). The hydroxamate additionally makes hydrogen-bonding interactions with nearby H142, H143, and Y306, all of which are important for enzyme activity and largely conserved through the HDAC family (Somoza et al., 2004). Crystal structures of both substrate and inhibitor complexes also reveal the importance of D101, which functions in substrate binding (Vannini et al., 2007; Dowling et al., 2008). The bindinginduced conformational changes of D101 are further propagated through the protein, and consequential changes are

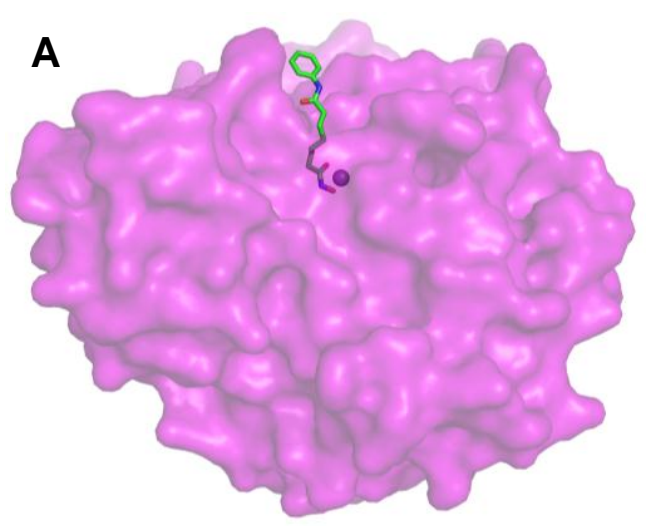

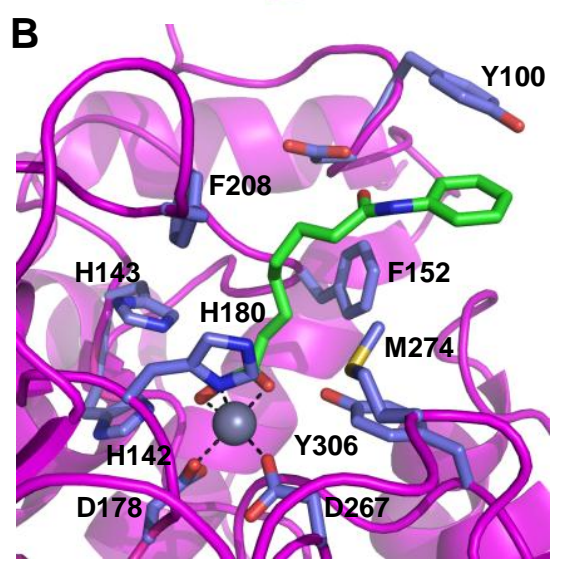

Figure 1. (A) Transparent surface view of HDAC8 complexed with SAHA showing the location of the active site pocket (PDB 1T69). (B) Active site binding interactions with SAHA showing the square pyramidal coordination geometry of the catalytic zinc by H180, D178, D267, and the HDACi. Other relevant residues that make bonding interactions with the inhibitor are shown in blue. seen specifically in L31 and Y111 (Dowling et al., 2008).

While most of the current HDACi are pan-inhibitors and bind to the catalytic metal ion in the different HDAC isoforms, many cellular processes (unrelated to tumorigenesis) rely on 
HDAC activity. Such broad-spectrum binding often results in the dysfunction of normal pathways, and can lead to various side effects, including fatigue, nausea, and diarrhea. Other serious side effects may also include thrombocytopenia and cardiac problems. Knockouts of HDACs 3, 5, and 9 caused severe cardiac complications in mice, whereas knockouts of any of the Class I HDACs have shown to be lethal in embryonic development or shortly after birth (Chang et al., 2004; Montgomery et al., 2008; Haberland et al., 2009). The design of isoformspecific inhibitors may help to eliminate such side effects and lead to the development of better cancer therapeutics. This topic has been nicely reviewed (Balasubramanian et al., 2009).

Further analysis of HDAC8-inhibitor crystal structures revealed that 3 specific residues (F152, F208, and M274) undergo conformational changes in HDAC8 (KrennHrubec et al., 2007). These residues, along with H180, G151, and Y306 form the walls of the active site tunnel; they are primarily hydrophobic and largely conserved throughout the Class I enzymes. The crystal structures show that when an inhibitor with a bulky (i.e. aryl) linker binds to the enzyme, F152 rotates away from M274, opening a unique, HDAC8-specific subpocket. In order to further exploit this subpocket, a set of 6 'linkerless' hydroxamic acid HDACi were rationally designed and synthesized (Figure 2) (KrennHrubec et al., 2007). These compounds were indeed potent inhibitors of HDAC8, and demonstrated selectivity for HDAC8 over HDACs 1 and 6 . A recent study
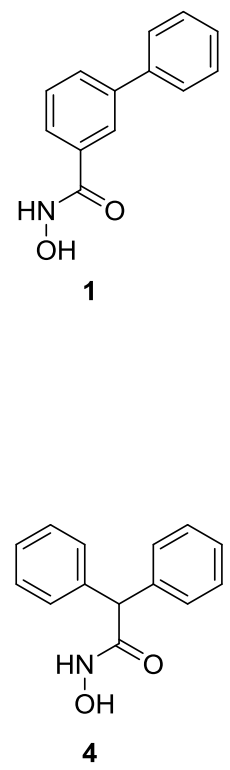

Figure 2. Structures of 6 'linkerless' hydroxamic acid HDACi (KrennHrubec et al., 2007).
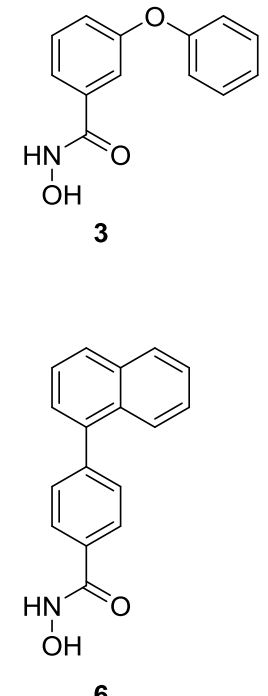
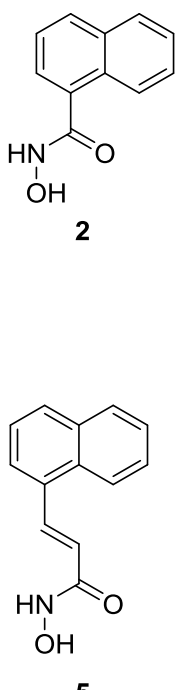
verified the use of Compound 2 for the selective inhibition of HDAC 8 in the treatment of neuroblastoma cell growth (Rettig et al., 2015). Compound 2 was well tolerated in mouse models, while broad-spectrum Vorinostat caused severe side effects, including diarrhea and weight loss. There has been no structural information to describe the mode of binding of these 'linkerless' inhibitors, however, or confirm the formation of the subpocket. Herein, we report the X-ray crystal structure of HDAC8 complexed with Compound 6 to $1.98 \AA$ A resolution. Using this structure as a model, we also describe the use of molecular docking studies to predict the binding interactions between HDAC8 and the 5 related hydroxamic acid HDACi.

\section{Materials and Methods}

HDAC8 Expression and Purification. Wild-type HDAC8 was recombinantly expressed in BL21(DE3) E. coli cells and purified according to published procedures (Cole et al., 2011). Briefly, overnight cultures were grown in LB media supplemented with ampicillin (AMP, final concentration $50 \mu \mathrm{g} / \mathrm{L}$ ). $50 \mathrm{~mL}$ of culture were used to inoculate minimal media supplemented with $1 \mathrm{mM}$ AMP, $2 \mathrm{mM} \mathrm{MgSO} 4,0.1 \mathrm{mM} \mathrm{CaCl}_{2}$, and 4 g glucose (per $1 \mathrm{~L}$ of media). Cells were grown for $\sim 2.5$ hours at $37{ }^{\circ} \mathrm{C}$ and $250 \mathrm{rpm}$ shaking, and induced by isopropyl $\beta$-D-1thiogalactopyranoside (IPTG, final concentration $0.4 \mathrm{mM}$ ) and $\mathrm{ZnCl}_{2}$ (final concentration 1 $\mathrm{mM})$. Cells were grown overnight at $18{ }^{\circ} \mathrm{C}$ and $250 \mathrm{rpm}$ shaking, and pelleted by centrifugation

$\left(4{ }^{\circ} \mathrm{C}, 6,000 \mathrm{rpm}, 10\right.$ minutes). The cell lysate was purified using affinity chromatography (Talon resin; Buffer A: 50 mM Tris, $500 \mathrm{mM} \mathrm{KCl,} 3$ mM $\beta$-mercaptoethanol, pH 8.0; Buffer B: $50 \mathrm{mM}$ Tris, $500 \mathrm{mM} \mathrm{KCl,} 250 \mathrm{mM}$ imidazole, $3 \mathrm{mM} \beta$-mercaptoethanol, $\mathrm{pH}$ 8.0), followed by size exclusion chromatography (50 mM Tris, $150 \mathrm{mM} \mathrm{KCl,} 1 \mathrm{mM}$ dithiothreitol (DTT), $\mathrm{pH}$ 8.0). 
Protein concentration was determined by measuring the absorbance at $280 \mathrm{~nm}\left(\varepsilon=49,640 \mathrm{M}^{-1}\right.$ $\left.\mathrm{cm}^{-1}\right)$.

Crystallization and Data Collection. Rectangular crystals of the HDAC8-Compound 6 complex were obtained in 1-2 days using the hanging drop vapor diffusion method with the following conditions: $2 \mu \mathrm{L}$ of protein solution $[\sim 5 \mathrm{mg} / \mathrm{mL}$ HDAC8 $(50 \mathrm{mM}$ Tris, $\mathrm{pH} 8,150 \mathrm{mM} \mathrm{KCl}, 5 \%$ glycerol, $1 \mathrm{mM}$ DTT, $0.03 \mathrm{M}$ Gly3, $4 \mathrm{mM}$ tris(2-carboxyethyl)phosphine) (TCEP), and $2 \mathrm{mM}$ Compound 6)] were mixed with $2 \mu \mathrm{L}$ of precipitant solution [4\% PEG 3350, $50 \mathrm{mM}$ buffer (MES, pH 5.3)] and equilibrated against a $500 \mu \mathrm{L}$ reservoir of precipitant solution. Single crystals were harvested and flash-cooled in 20\% PEG 3350, 20\%, glycerol, and 0.1 M MES buffer (pH 5.3). Crystals diffracted X-rays to $1.98 \AA$ ̊ resolution at the Advanced Photon Source, beamline NE-CAT 24-ID-C (Argonne National Lab) using a PILATUS-6MF detector. Diffraction data were indexed and scaled using XDS as implemented in the Rapid Automated Processing of X-ray Data package (https://github.com/RAPD/RAPD). Crystals belonged to space group $P 2_{1}$ with unit cell dimensions $a=53.44 \AA, b=84.56 \AA, c=94.32 \AA$.

Structure Determination and Refinement. The structure was solved by using PHASER as implemented in RAPD (https://github.com/RAPD/RAPD) with the atomic coordinates of HDAC8 complexed with substrate (PDB code: 3EZT, less ions, solvent, and substrate) as a search probe in rotation and translation function calculations. Iterative cycles of refinement and model building were performed with Phenix (Adams et al., 2002) and Coot (Emsely and Cowtan, 2004), respectively, to improve the structure as monitored by $R_{\text {free. }}$ The atomic coordinates for Compound 6, including 2 crystallographic (non-biological) inhibitors, a disordered PEG 
molecule, and solvent molecules were added during the final stages of refinement. Disordered segments in the final model include M1-Q12 and E379-H389 in monomer A, and M1-Q12 and I378-H389 in monomer B. The final Ramachandran plot indicated that $98 \%$ of the residues adopted preferred conformations, $2 \%$ were allowed, and $0 \%$ were in outlier conformations. Data collection and refinement statistics are recorded in Table 1 (Supporting Information). The atomic coordinates and structure factors of the HDAC8-Compound 6 complex have been deposited in the Protein Data Bank (www.rcsb.org) with accession code 5FCW.

Molecular Docking Studies. Inhibitor docking studies were performed using AutoDock Vina (Trott and Olson, 2010). The 6 hydroxamic acid HDACi were first energy-minimized and converted to .pdb files using ChemBioDraw Ultra 14.0 and Chem3D Pro 14.0 (CambridgeSoft). Chain A of the HDAC8-Compound 6 complex (less the potassium ions, solvent, and the inhibitor) was used as the model; the catalytic zinc was included in the model. Residues F152, F208, and M274 were defined as flexible residues. The search space was defined as following: center_x $=37.141$, center_y $=11.218$, and center_z $=120.589$, with size_x $=54$, size_y $=38$, size $\_z=48$, with the spacing set to $0.375 \AA$ and the exhaustiveness set to 25 iterations. The .pdbqt output files were analyzed in Pymol, which was compiled from an open source.

\section{Results and Discussion}

\section{Structural Studies}

Most of the current HDACi are pan-inhibitors and non-selectively inhibit a number of HDAC isoforms. Because the isoforms have distinct cellular functions, however, and the activities of some isoforms may actually be beneficial in cancer treatments, the need for selective 
inhibition is crucial (Balasubramanian, 2009). Using a set of HDACi that were designed to target a unique subpocket in HDAC8, we report the first X-ray crystal structure of Compound 6 complexed with HDAC8 to $1.98 \AA$ A resolution (Figure 3). The hydroxamic acid of the inhibitor chelates the catalytic zinc ion in a bidentate fashion as expected, and the active site residues (D178, H180, and D267) complete the metal coordination sphere. As in many of the other HDAC8-inhibitor complexes, the inhibitor makes hydrogen bonding interactions with H142,

H143, D178, H180, D267, and Y306, and van der Waals interactions with F152 and F208.

A

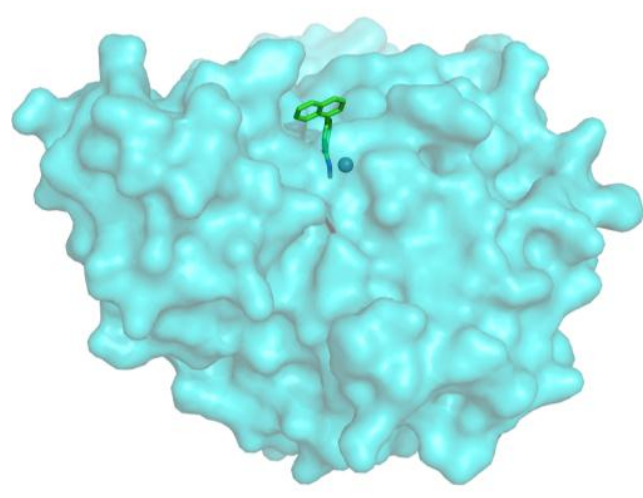

B

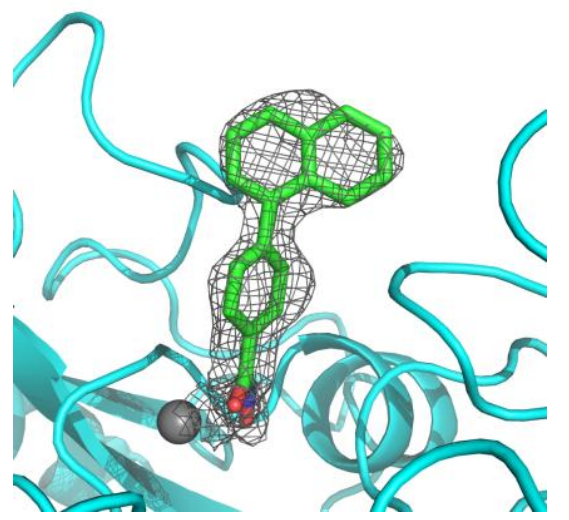

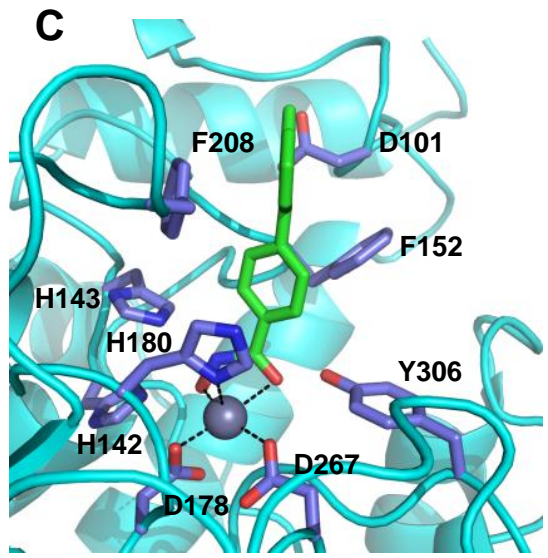

Figure 3. (A) Transparent surface view of HDAC8 complexed with Compound 6 showing the location of the active site pocket. (B) Omit map confirming inhibitor binding, contoured to 1.0 sigma (C) Metal binding interactions of Compound 6 in active site pocket.

The inhibitor also makes van der Waals interactions with P273 on chain B (not shown), and one of the crystallographic inhibitors. There are 2 crystallographic inhibitors that lie at the interface of the HDAC8 monomers. They do not bind in the enzyme and, therefore, do not appear to be biological. Instead, these inhibitors construct a network of van der Waals interactions; they make van der Waals interactions with themselves and nearby water

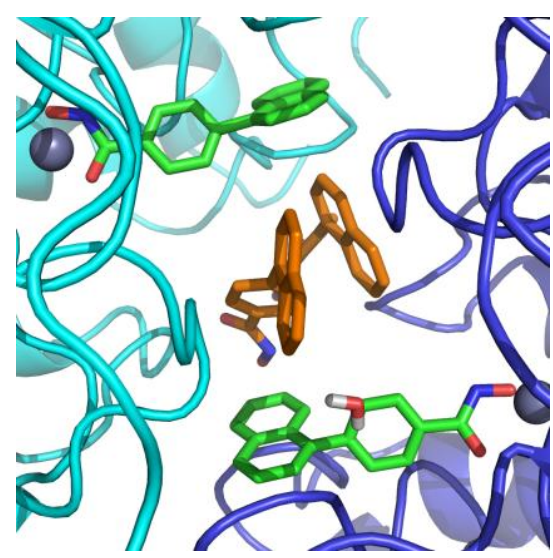

Figure 4. Van der Waals interactions between biological (green) and nonbiological (orange) inhibitors. Dashed lines are omitted for clarity. 
molecules, as well as the zinc-binding (biological) inhibitors and select residues (Figure 4).

These interactions facilitate the in vitro dimerization that is commonly required for HDAC8 crystallization.

More importantly, this structure is the first to confirm the formation of the predicted HDAC8-specific subpocket using the rationally designed isoform-specific inhibitors. In the enzymesubstrate structure, F152 and M274 point towards one another, making van der Waals interactions and forming one wall of the active site pocket (Figure 5A). In our structure, however, the aryl linker of the inhibitor splits these residues, causing F152 to rotate away from M274 (Figure 5B). This slight rotation creates the HDAC8-unique subpocket, which may be further exploited for enhanced isoform-specific inhibition.

Interestingly, recent studies with HDAC8 from the parasite Schistosoma mansoni (smHDAC8) have been seeking to identify similarities and, more importantly, differences between human HDAC8 and smHDAC8 for the development of new anti-parasitic drugs. The recent crystal structure of smHDAC8 reveals that F152 (smHDAC8 numbering F151) maintains a 'flipped-out' conformation compared to human HDAC8 (Marek, et al., 2013). This conformation creates a larger active site which should accommodate larger inhibitors compared to human HDAC8 and, therefore, offers the

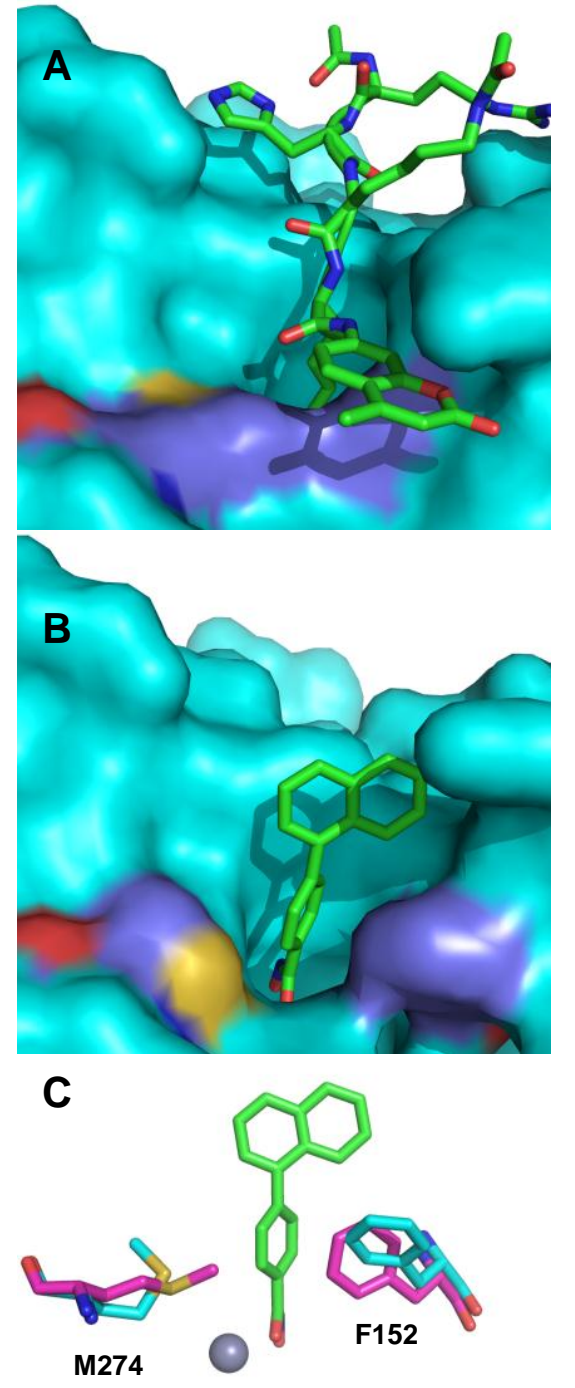

Figure 5. (A) In the HDAC8-substrate complex (2V5W), F152 and M274 interact to form a wall of the active site. (B) In the HDAC8-Compound 6 structure, F152 rotates away from M274 forming the unique subpocket. The rotation of M274 is highlighted in (C) with the HDAC8-Compound 6 structure shown in cyan and the HDAC8-substrate complex shown in magenta. 
possibility for organism-specific inhibition. Indeed, smHDAC8 has been inhibited by the traditional HDACi SAHA and M344, as well as bulky 'linkerless' hydroxamate inhibitors (Kannan, et al., 2014; Heimburg, et al., 2016).

\section{Docking Studies}

Because crystallization of HDAC8 requires a ligand (substrate or inhibitor), and the other enzyme-inhibitor complexes failed to crystallize under a variety of conditions, molecular docking studies were used to visualize the binding of the 5 remaining HDACi. Docking studies were performed using AutoDock Vina (Trott and Olson, 2010). The use of Autodock Vina and similar molecular docking programs has been described previously (Hou, et al., 2015, Pottel et al., 2014, Sixto-López et al., 2014). Chain A of the HDAC8-Compound 6 complex was used as the model, and residues F152, F208, and M274 were defined as flexible. The most favorable (lowest energy) docking result with Compound 6 aligns well with our X-ray crystal structure (Supporting Information). The most favorable docking outputs for all 6 HDACi are shown in Figure 6. It is important to note that AutoDock Vina is programmed for 4-coordinate metal binding, and will not accurately predict bidentate metal chelation; the expected bidentate chelation was instead confirmed in the crystal structure.

Compound 6 is predicted to be the strongest binder, followed by Compound 5 . These results correlate nicely with the reported activity assays; these 2 inhibitors were the most potent against HDAC8 activity, with $\mathrm{IC}_{50}$ values of 0.3 and $0.7 \mu \mathrm{M}$, respectively (KrennHrubec et al., 2007). Compounds 5 and 6 have the longest 'linkers' of the set, allowing them to best bind the catalytic zinc ion and make interactions with the mouth of the active site. Compounds 3 and 4 have similar binding energies, but very different $\mathrm{IC}_{50}$ values $(6.6$ and $66.0 \mu \mathrm{M}$, respectively). 
While they both appear to bind to the catalytic zinc ion, Compound 4 is more sterically hindered about the alpha carbon, which may hinder its binding in the active site. While the docking studies predict that neither Compounds 1 nor 2 bind to the catalytic zinc ion, both have modest $\mathrm{IC}_{50}$ values (20.0 and $14.0 \mu \mathrm{M}$, respectively), and Compound 2 was recently used to inhibit the growth of neuroblastoma cells, as discussed above (Rettig et al., 2015).
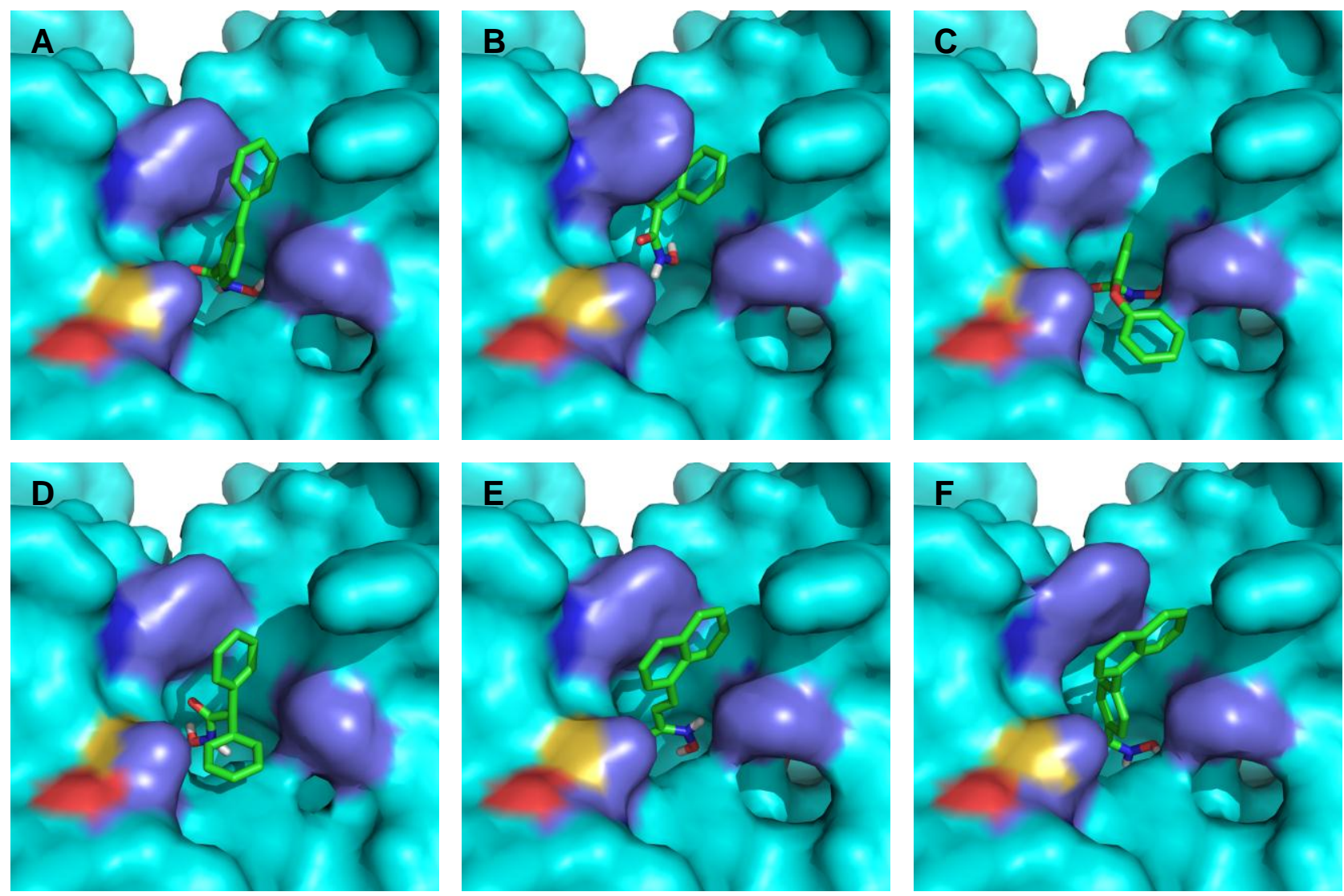

Figure 6. Predicted inhibitor binding from most favorable docking results. Panel A-F correspond to Compounds 16 , respectively.

\section{Conclusions}

In conclusion, the X-ray crystal structure of HDAC8 complexed with Compound 6 confirms the formation of a unique subpocket in HDAC8. The bulky aryl ring of the inhibitor causes F152 and M274 to split, which exposes the subpocket. Our docking studies suggest that the longer 'linker' of Compounds 5 and 6 allow these inhibitors to both chelate the catalytic zinc 
and make interactions with the mouth of the active site. As such, these inhibitors exhibit the tightest binding and also the strongest potency (KrennHrubec et al., 2007). Further exploitation of this subpocket may lead to the design and development of new isoform-specific inhibitors.

\section{Acknowledgements}

This work is based upon research conducted at the Advanced Photon Source on the Northeastern Collaborative Access Team beamlines, which are supported by a grant from the National Institute of General Medical Sciences (P41 GM103403) of the National Institutes of Health. The Pilatus 6M detector on 24-ID-C beam line is funded by a NIH-ORIP HEI grant (S10 RR029205). Use of the Advanced Photon Source, an Office of Science User Facility operated for the US Department of Energy (DOE) Office of Science by Argonne National Laboratory, was supported by the US DOE under Contract No. DE-AC02-06CH11357. KEC would like to thank Ithaca College and Christopher Newport University for funding. Undergraduate summer research was supported by the Dana Award (RF, Ithaca College) and the Summer Scholars Program (AT, Christopher Newport University). We would also like to thank Dr. Scott Ulrich for providing the inhibitors, and Dr. Daniel Dowling for helpful conversations. 


\section{References}

Adams, P. D., Grosse-Kunstleve, R. W., Hung, L. W., Ioerger, T. R., McCoy, A. J., Moriarty, N. W., Read, R. J., Sacchettini, J. C., Sauter, N. K., and Terwilliger, T. C. (2002) Acta Crystallogr. Sect. D: Biol. Crystallogr., 58, 1948-1954.

Balasubramanian, S., Verner, E., Buggy, J. J. (2009). Isoform-Specific Histone Deacetylase Inhibitors: The Next Step? Cancer Letters, 280, 211-221.

Bolden, J. E., Peart, M. J., Johnstone, R. W. (2006) Anticancer Activities of Histone Deacetylase Inhibitors. Nat. Rev., 5, 769-784.

Chang, S., McKinsey, T.A., Zhang, C.L., Richardson, J.A., Hill, J.A. and Olson, E.N. (2004) Histone Deacetylases 5 and 9 Govern Responsiveness of the Heart to a Subset of Stress Signals and Play Redundant Roles in Heart Development. Mol. Cell. Bio., 24, 8467-8476.

Cole, K.E., Dowling, D.P., Boone, M.A., Phillips, A.J., Christianson, D.W. (2011) Structural Basis of the Antiproliferative Activity of Largazole, a Depsipeptide Inhibitor of the Histone Deacetylases. J. Am. Chem. Soc., 32, 12474-12477.

De Ruijter, A.J., van Gennip, A.H., Caron, H.N., Kemp, S., van Kuilenburg, A.B. (2003) Histone Deacetylases (HDACs): Characterization of the Classical HDAC Family. Biochem. J., 370, 737749.

Dowling, D.P., Gantt, S.L., Gattis, S.G., Fierke, C.A., Christianson, D.W. (2008) Structural Studies of Human Histone Deacetylase 8 and its Site-Specific Variants Complexed with Substrate and Inhibitors. Biochemistry, 47, 13554-13563.

Dowling, D.P., Gattis, S.G., Fierke, C.A., Christianson, D.W. (2010) Structures of MetalSubstituted Human Histone Deacetylase 8 Provide Mechanistic Inferences on Biological Function. Biochemistry, , 49, 5048-5056.

Emanuele S., Lauricella M., Tesoriere, G. (2008) Histone Deacetylase Inhibitors: Apoptotic Effects and Clinical Implications. Int. J. Oncol., 33, 637-646.

Emsely, P. and Cowtan, K. (2004) Acta Crystallogr. Sect. D: Biol. Crystallogr., 60, 2126-2132.

Fenichel, M.P. (2015) FDA Approves New Agent for Multiple Myeloma. J. Natl. Cancer Inst., 107, 6-7.

Glozak, M.A., Seto, E. (2007) Histone Deacetylases and Cancer. Oncogene., 26, 5520-5432.

Guan, P., Fang, H. (2010) Clinical Development of Histone Deacetylase Inhibitor Romidepsin. Drug Disc. Ther., 4, 388-391. 
Haberland, M., Montgomery, R.L., Olson, E.N. (2009) The Many Roles of Histone Deacetylases in Development and Physiology: Implications for Disease and Therapy. Nature Rev. Gen., 10, $32-42$.

Heimburg, T., Chakrabarti, A., Lancelot, J., Marek, M., Melesina, J., Hauser, A.T., Shaik, T.B., Duclaud, S., Robaa, D., Erdmann, F. and Schmidt, M. (2016) Structure-Based Design and Synthesis of Novel Inhibitors Targeting HDAC8 from Schistosoma mansoni for the Treatment of Schistosomiasis. J. Med. Chem., 59, 2423-2435.

Hou, X., Dr, J., Renshuai, L., Zhou, Y., Li, M., Xu, W., Fang, H. (2015) Enhancing the Sensitivity of Pharmacophore-Based Virtual Screening by Incorporating Customized ZBG Features: A Case Study Using Histone Deacetylase 8. J. Chem. Inf. Model, 55, 861-871.

Huang, L., Pardee, A.B. (2000) Suberoylanilide Hydroxamic Acid as a Potential Therapeutic Agent for Human Breast Cancer Treatment. Mol. Med., 6, 849-66.

Kannan, S., Melesina, J., Hauser, A.T., Chakrabarti, A., Heimburg, T., Schmidtkunz, K., Walter, A., Marek, M., Pierce, R.J., Romier, C. and Jung, M. (2014) Discovery of Inhibitors of Schistosoma mansoni HDAC8 by Combining Homology Modeling, Virtual Screening, and In Vitro Validation. J. Chem. Inf. Model, 54, 3005-3019.

KrennHrubec, K., Marshal, B.L., Hedglin, M., Verdin, E., Ulrich, S.M. (2007) Design and Evaluation of 'Linkerless' Hydroxamic Acids as Selective HDAC8 Inhibitors. Bioorg. Med. Chem. Lett., 17, 2874-2978.

Lee, H.Z., Kwitkowski, V.E., Del Valle, P.L., Ricci, M.S., Saber, H., Habtemariam, B.A., Bullock, J., Bloomquist, E., Shen, Y.L., Chen, X.H., Brown, J., Mehrotra, N., Dorff, S., Charlab, R., Kane, R.C., Kaminskas, E., Justice, R., Farrell, A.T., Pazdur, R. (2015) FDA Approval: Belinostat for the Treatment of Patients with Relapsed or Refractory Peripheral T-cell Lymphoma. Clin. Cancer Res., 21, 2666-2670.

Mann, B.S., Johnson, J. R., Cohen, M. H., Justice, R., Pazdur, R. (2007) FDA Approval Summary: Vorinostat for Treatment of Advanced Primary Cutaneous T-cell Lymphoma. The Oncologist, 12, 1247-1252.

Marek, M., Kannan, S., Hauser, A.T., Mourao, M.M., Caby, S., Cura, V., Stolfa, D.A., Schmidtkunz, K., Lancelot, J., Andrade, L. and Renaud, J.P. (2013) Structural Basis for the Inhibition of Histone Deacetylase 8 (HDAC8), a Key Epigenetic Player in the Blood Fluke Schistosoma mansoni. PLoS Pathog, 9, e1003645.

Mei, S., Ho, A. D., Mahlknecht, U. (2004) Role of Histone Deacetylase Inhibitors in the Treatment of Cancer. Int. J. Oncol., 25, 1509-1519.

Montgomery, R.L., Potthoff, M.J., Haberland, M., Qi, X., Matsuzaki, S., Humphries, K.M., Richardson, J.A., Bassel-Duby, R. and Olson, E.N. (2008) Maintenance of Cardiac Energy Metabolism by Histone Deacetylase 3 in Mice. J. Clin. Invest., 118, 3588-3597. 
Pottel, J., Therrien, E., Gleason, J.L., Moitessier, N. (2014) Docking Ligands into Flexible and Solvated Macromolecules. 6. Development and Application to the Docking of HDACs and Other Zinc Metalloenzymes Inhibitors. J. Chem. Inf. Model. 54, 254-65.

Rettig, I., Koeneke, E., Trippel, F., Mueller, W.C., Burhenne, J., Kopp-Schneider, A., Fabian, J., Schober, A., Fernekorn, U., von Deimling, A. and Deubzer, H.E. (2015) Selective Inhibition of HDAC8 Decreases Neuroblastoma Growth In Vitro and In Vivo and Enhances Retinoic AcidMediated Differentiation. Cell Death Dis., 6, 1657.

Sixto-López, Y., Gómez-Vidal, J.A. Correa-Basurto, J. (2014) Exploring the Potential Binding Sites of Some Known HDAC Inhibitors on Some HDAC8 Conformers by Docking Studies. Appl. Biochem. Biotechnol. 173, 1907-926.

Somoza, J.R., Skene, R.J., Katz, B.A., Mol, C., Ho, J.D., Jennings, A.J., Luong, C., Arvai, A., Buggy, J.J., Chi, E., Tang, J., Sang, B.C., Verner, E., Wynands, R., Leahy, E.M., Dougan, D.R., Snell, G., Navre, M., Knuth, M.W., Swanson, R.V., McRee, D.E., Tari, L.W. (2004) Structural Snapshots of Human HDAC8 Provide Insights into the Class I Histone Deacetylases. Structure, 12, 1325-1334.

Trott, O., Olson, A.J. (2010) AutoDock Vina: Improving the Speed and Accuracy of Docking with a New Scoring Function, Efficient Optimization and Multithreading. J. Comput. Chem. 31, 455-461.

Vannini, A., Volpari, C., Filocamo, G., Casavola, E.C., Brunetti, M., Renzoni, D., Chakravarty, P., Paolini, C., De Francesco, R., Gallinari, P., Steinkühler, C., Di Marco, S. (2004) Crystal Structure of a Eukaryotic Zinc-Dependent Histone Deacetylase, Human HDAC8, Complexed with a Hydroxamic Acid Inhibitor. Proc. Natl. Acad. Sci., 101,15064-15069.

Vannini, A., Volpari, C., Gallinari, P., Jones, P., Mattu, M., Carfi, A., De Francesco, R., Steinkühler, C., Di Marco, S. (2007) Substrate Binding to Histone Deacetylase As Shown by the Crystal Structure of the HDAC8-Substrate Complex. EMBO Reports, 8, 879-884. 